\title{
Precision Measurements of Collective Oscillations in the BEC-BCS Crossover
}

\author{
A. Altmeyer ${ }^{1}$ S. Riedl, ${ }^{1,2}$ C. Kohstall,${ }^{1}$ M. J. Wright,${ }^{1}$ R. Geursen,${ }^{1}$ \\ M. Bartenstein, ${ }^{1}$ C. Chin,${ }^{3}$ J. Hecker Denschlag, ${ }^{1}$ and R. Grimm ${ }^{1,2}$ \\ ${ }^{1}$ Inst. of Experimental Physics and Center for Quantum Physics, Univ. Innsbruck, 6020 Innsbruck, Austria \\ ${ }^{2}$ Inst. for Quantum Optics and Quantum Information, Acad. of Sciences, 6020 Innsbruck, Austria \\ ${ }^{3}$ James Franck Institute, Physics Department of the University of Chicago, Chicago, IL 60637, USA
}

(Dated: August 8, 2018)

\begin{abstract}
We report on precision measurements of the frequency of the radial compression mode in a strongly interacting, optically trapped Fermi gas of ${ }^{6} \mathrm{Li}$ atoms. Our results allow for a test of theoretical predictions for the equation of state in the BEC-BCS crossover. We confirm recent quantum MonteCarlo results and rule out simple mean-field BCS theory. Our results show the long-sought beyondmean-field effects in the strongly interacting BEC regime.
\end{abstract}

PACS numbers: 34.50.-s, 05.30.Fk, 39.25.+k, 32.80.Pj

Ultracold, strongly interacting Fermi gases [1, 2, 3, 4, 5, 6, 7, 8, 9, 10, 11, 12, 13] have attracted considerable attention over the past few years, serving as unique model systems to create, control, and investigate novel states of quantum matter. Experimentally, the availability of such systems has opened up exciting possibilities to study many-body quantum phenomena like molecular Bose-Einstein condensation (BEC) 3] and the crossover from BEC to a Bardeen-Cooper-Schrieffer (BCS) type superfluid [4, 5, 6, 7, 8, 9, 10, 11, 12, 13]. These experiments may also lead to a better understanding of strongly interacting quantum systems in different areas of physics, ranging from high- $\mathrm{T}_{c}$ superconductors to neutron stars and the quark-gluon plasma.

A degenerate two-component Fermi gas undergoes the BEC-BCS crossover 14] when the $s$-wave scattering length $a$ is varied from positive to negative values across a scattering resonance. In the crossover region, where $a$ is comparable with or larger than the interparticle spacing, the equation of state is governed by many-body effects. Understanding the equation of state is a fundamentally important challenge and constitutes a difficult task for many-body quantum theories, even in the zerotemperature limit. Mean-field BCS theory [14] provides a reasonable interpolation between the well-understood limits. More sophisticated crossover approaches [15] yield quantitatively different results in certain regimes, none of them however providing a complete description of the problem. The most advanced theoretical results were obtained by numerical calculations based on a quantum Monte-Carlo (QMC) approach [16].

On the BEC side of the crossover, there is an interesting competition in the equation of state between the strong interactions in a Bose gas and the onset of fermionic behavior. For a strongly interacting Bose gas, one can expect quantum depletion to increase the average energy per particle. To lowest order, this beyond-meanfield effect leads a correction to the equation of state predicted by Lee, Huang, and Yang (LHY) almost 50 years ago [17]. Beyond mean-field effects are expected to reduce the compressibility of a strongly interacting Bose gas as compared to the weakly interacting case. How- ever, when approaching the resonance, fermionic behavior emerges and the system loses its purely bosonic character, which increases the compressibility of the strongly interacting gas. Mean-field BCS theory does not contain beyond-mean field effects and the LHY correction is absent there. However, the QMC results predict beyondmean-field effects to be visible on the BEC-side of the crossover [16].

In this Letter, we report on precision measurements of the radial compression mode in an optically trapped, strongly interacting Fermi gas of ${ }^{6} \mathrm{Li}$ atoms. The mode serves as a sensitive probe for the compressibility and thus the equation of state of a superfluid gas in the BECBCS crossover [18, 19]. We reach a precision level that allows us to distinguish between the predictions resulting from mean field BCS theory and QMC calculations. Previous experiments on collective modes, performed at Duke University [8, 11] and at Innsbruck University [9], showed frequency changes in the BEC-BCS crossover in both the slow axial mode and the fast radial compression mode of a cigar-shaped sample. The accuracy, however, was insufficient for a conclusive test of the different manybody theories in the strongly interacting regime.

We prepare a strongly interacting, degenerate gas of ${ }^{6} \mathrm{Li}$ atoms in the lowest two internal states as described in our previous publications [4, 9, 10]. The broad Feshbach resonance centered at a magnetic field of $B=834 \mathrm{G}$ facilitates precise tuning of the scattering length $a$ [20]. Forced evaporative cooling is performed in a 1030-nm near-infrared laser beam focussed to a waist of $54 \mu \mathrm{m}$ at $764 \mathrm{G}$. This results in a deeply degenerate cloud of $N=2.0(5) \times 10^{5}$ atoms. By adiabatically increasing the trap laser power after cooling, the sample is recompressed to achieve nearly harmonic confinement. In the axial direction the gas is magnetically confined in the curvature of the field used for Feshbach tuning with an axial trap frequency of $\omega_{z} / 2 \pi=22.4 \mathrm{~Hz}$ at $834 \mathrm{G}$. The experiments reported here are performed at two different final values of the laser power of the recompressed trap. At $135 \mathrm{~mW}(540 \mathrm{~mW})$, the trap is $1.8 \mu \mathrm{K}(7.3 \mu \mathrm{K})$ deep and the radial trap frequency is $\omega_{r} \approx 2 \pi \times 290 \mathrm{~Hz}(590 \mathrm{~Hz})$. The Fermi energy of a non-interacting cloud is calcu- 


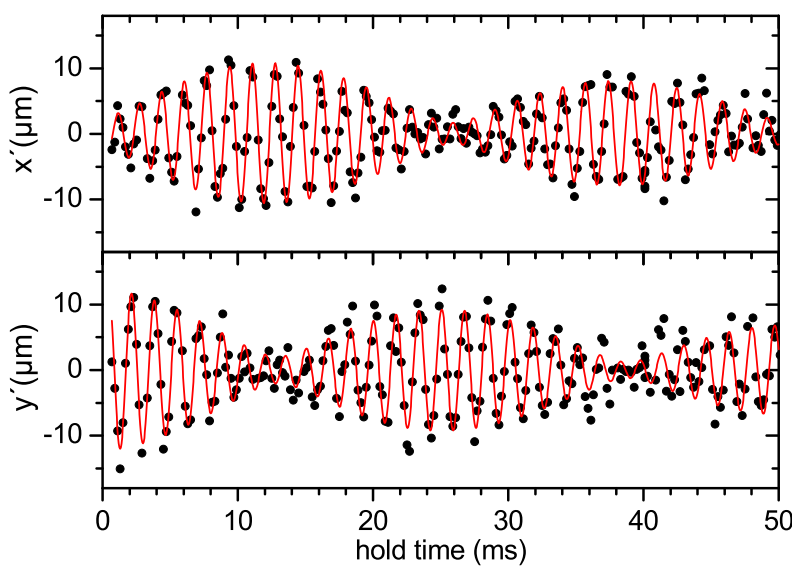

FIG. 1: Radial sloshing observed at a trap power of $540 \mathrm{~mW}$ and $B=735 \mathrm{G}\left(1 / k_{F} a=1.55\right)$. The two-dimensional centerof-mass motion is represented in a coordinate system $\left(x^{\prime}, y^{\prime}\right)$ rotated by $45^{\circ}$ with respect to the principal axes of the trap. The beat signal between the two sloshing eigenmodes demonstrates the ellipticity of the trap with the two eigenfrequencies $\omega_{x} / 2 \pi=570 \mathrm{~Hz}$ and $\omega_{y} / 2 \pi=608 \mathrm{~Hz}$ (ellipticity $\epsilon=0.066$ ).

lated to $E_{F}=\hbar^{2} k_{F}^{2} / 2 m=\hbar\left(3 \omega_{r}^{2} \omega_{z} N\right)^{1 / 3}=k_{B} \times 500 \mathrm{nK}$ $(800 \mathrm{nK})$; here $m$ is the mass of an atom and $k_{B}$ is Boltzmann's constant.

Since our first measurements on collective excitation modes [9], we have upgraded our apparatus with a twodimensional acousto-optical deflection system for the trapping beam and a new imaging system along the trapping beam axis. These two improvements provide us with full access to manipulate and observe the radial motion.

The trap beam profile is somewhat elliptic because of imperfections and aberrations in the optical set up. To simultaneously excite the two eigenmodes of the radial sloshing motion, we initially displace the trapped sample into a direction between the horizontal and vertical principal axes of the radial potential. After a variable hold time, during which the cloud oscillates freely, we turn off the optical trap. After a time of flight of typically $4 \mathrm{~ms}$ we take an absorption image of the released cloud. The center-of-mass position of the cloud then reflects its momentum at the instant of release. The experimental results in Fig. 1 demonstrate the sloshing with a beat between the two eigenmodes. A careful analysis of such data 21] allows us to determine the eigenfrequencies $\omega_{x}$ (horizontal sloshing) and $\omega_{y}$ (vertical sloshing) to within a relative uncertainty of typically $2 \times 10^{-3}$. We finally derive the mean sloshing frequency $\omega_{\perp}=\sqrt{\omega_{x} \omega_{y}}$ and the ellipticity parameter $\epsilon=\left(\omega_{y}-\omega_{x}\right) / \omega_{\perp}$.

To excite the radial compression oscillation we reduce the trap light power for a short time interval of $\sim 100 \mu \mathrm{s}$, inducing an oscillation with a relative amplitude of typically $10 \%$. After a variable hold time the cloud is released from the trap. From fits of two-dimensional ThomasFermi profiles to images of the expanding cloud taken $4 \mathrm{~ms}$ after release, we determine the mean cloud radius.

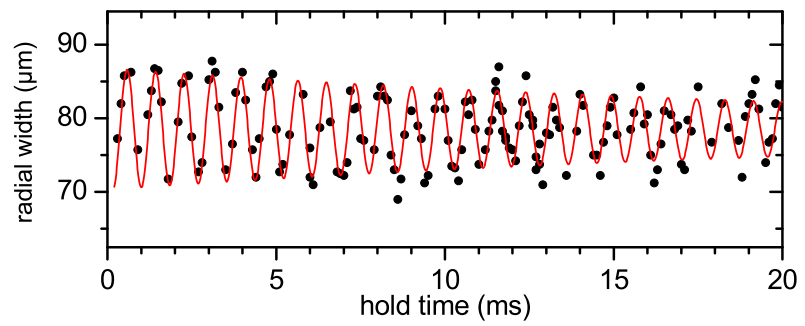

FIG. 2: Radial compression oscillation observed for the same conditions as the sloshing mode data in Fig. 1] The radial width is determined by averaging the horizontal and vertical Thomas-Fermi radii after expansion. Here we obtain $\omega_{c} / 2 \pi=$ $1185 \mathrm{~Hz}$.

A typical set of measurements is shown in Fig. 2, A fit of a damped harmonic oscillation to such data yields the frequency $\omega_{c}$ and damping rate $\gamma$ of the radial compression mode.

Our experiments are performed close to the limit of an elongated harmonic trap potential with cylindrical symmetry. This elementary case is of great general relevance for many quantum gas experiments in optical and magnetic traps (see, e.g., 22] ), and collective excitations are conveniently normalized to the trap frequency $\omega_{r}[18,19]$. The compression mode frequency can then be written as $\omega_{c}=f_{c} \omega_{r}$, where $f_{c}$ is a dimensionless function of the interaction parameter $1 / k_{F} a$ and is related to an effective polytropic index $\Gamma[18,19]$ of the equation of state by $\omega_{c}^{2}=2(\Gamma+1) \omega_{r}^{2}$.

In order to compare our experimental results with theory, we consider the quantity $f_{c}$, i.e. the normalized compression mode frequency of the ideal, cylindrically symmetric, elongated trap. We find, that for our experimental conditions, $f_{c}$ is approximated by the ratio $\omega_{c} / \omega_{\perp}$ of the measured compression mode $\left(\omega_{c}\right)$ and mean sloshing mode $\left(\omega_{\perp}\right)$ frequencies to better than one percent. On the desired accuracy level of $10^{-3}$, however, two small effects have to be taken into account: the residual trap ellipticity and the anharmonicity of the radial potential in combination with the spatial extension of the trapped sample. We thus introduce two small corresponding corrections, expressing $f_{c}$ in the form $f_{c}=$ $\left(1-\kappa \epsilon^{2}+b \alpha\right) \omega_{c} / \omega_{\perp}$.

For the ellipticity correction $\kappa \epsilon^{2}$, a straightforward solution of the hydrodynamic eigenfrequency equation 21] yields $\kappa=(2+\Gamma) / 4 \Gamma$, where $\Gamma$ can be approximated by $\Gamma=\left(\omega_{c} / \omega_{\perp}\right)^{2} / 2-1$. For the anharmonicity correction, the parameter $\alpha=\frac{1}{2} m \omega_{\perp}^{2} r_{\mathrm{rms}}^{2} / U_{0}$ relates the potential energy associated with the root-mean-square radius $r_{\text {rms }}$ 23] of the trapped cloud to the trap depth. The coefficient $b$ results from the differential anharmonicity shifts in the compression and sloshing modes and can be calculated according to 21, 24, 25]. We obtain [21] $b=0.167$ and 0.280 in the limits of BEC and unitarity, respectively.

Our measurements on the sloshing and compression modes are summarized in Table I, including the two small corrections. For the data in the strongly interacting 
TABLE I: Experimental data on radial collective modes in the BEC-BCS crossover. The data in the upper seven (lower eight) rows refer to the sets of measurements taken in the shallower (deeper) trap with $U_{0}=1.8 \mu \mathrm{K}$ and $E_{F}=500 \mathrm{nK}$ $\left(U_{0}=7.3 \mu \mathrm{K}\right.$ and $\left.E_{F}=800 \mathrm{nK}\right)$. The values in parentheses indicate $1 \sigma$ fit uncertainties of individual measurements. Note that a systematic scaling uncertainty of $\sim 4 \%$ for $1 / k_{F} a$ results from the uncertainty in the atom number $N=2.0(5) \times 10^{5}$.

\begin{tabular}{|cc|cc|cc|cc|}
\hline & & \multicolumn{2}{|c|}{ sloshing } & \multicolumn{2}{c|}{ compression } & \multicolumn{2}{c|}{ corr. } \\
$(\mathrm{G})$ & & $1 / k_{F} a$ & $\omega_{\perp} / 2 \pi$ & $\epsilon$ & $\omega_{c} / 2 \pi$ & $\gamma / \omega_{\perp}$ & \multicolumn{2}{c}{$\kappa \epsilon^{2} b \alpha$} \\
\hline 727.8 & 2.21 & $292.7(5)$ & $0.083(3)$ & $596.3(6)$ & $0.007(2)$ & 48 & 20 \\
735.1 & 1.96 & $298.6(5)$ & $0.091(3)$ & $602.8(8)$ & $0.008(3)$ & 60 & 26 \\
742.5 & 1.75 & $294.5(5)$ & $0.067(3)$ & $593.2(7)$ & $0.005(2)$ & 33 & 28 \\
749.8 & 1.55 & $296.3(4)$ & $0.073(3)$ & $599.0(7)$ & $0.006(2)$ & 38 & 28 \\
760.9 & 1.27 & $296.0(4)$ & $0.088(2)$ & $592.3(7)$ & $0.009(2)$ & 58 & 24 \\
771.9 & 1.03 & $293.6(7)$ & $0.074(5)$ & $586.2(8)$ & $0.007(3)$ & 41 & 27 \\
834.1 & 0 & $287.5(7)$ & $0.073(5)$ & $519.4(9)$ & $0.014(3)$ & 55 & 94 \\
\hline 757.2 & 1.07 & $605.0(9)$ & $0.065(3)$ & $1210.9(12)$ & $0.010(2)$ & 32 & 13 \\
768.2 & 0.87 & $592.5(7)$ & $0.069(2)$ & $1186.6(12)$ & $0.012(2)$ & 36 & 16 \\
775.6 & 0.75 & $590.2(4)$ & $0.060(1)$ & $1170.2(21)$ & $0.007(4)$ & 28 & 14 \\
782.2 & 0.64 & $604.8(9)$ & $0.061(3)$ & $1187.1(16)$ & $0.006(3)$ & 29 & 16 \\
801.3 & 0.38 & $586.8(7)$ & $0.063(2)$ & $1135.2(12)$ & $0.010(2)$ & 33 & 24 \\
812.3 & 0.24 & $586.5(7)$ & $0.058(2)$ & $1106.9(16)$ & $0.014(3)$ & 30 & 33 \\
834.1 & 0 & $596.3(9)$ & $0.070(3)$ & $1089.0(12)$ & $0.010(2)$ & 48 & 40 \\
849.1 & -0.14 & $583.2(7)$ & $0.052(2)$ & $1046.7(37)$ & $0.007(2)$ & 29 & 47 \\
\hline
\end{tabular}

BEC regime $\left(1 / k_{F} a \gtrsim 1\right)$ we used the weaker trap with $\omega_{\perp} / 2 \pi \approx 290 \mathrm{~Hz}$ to minimize unwanted heating by inelastic collisions. Closer to resonance $\left(1 / k_{F} a \lesssim 1\right)$ inelastic processes are strongly suppressed, but the increasing cloud size introduces larger anharmonicity shifts. Here we chose the deeper trap with $\omega_{\perp} / 2 \pi \approx 590 \mathrm{~Hz}$. On the BCS side of the resonance we observed increased damping as a precursor of the breakdown of hydrodynamics [9, 11]. We thus restricted our measurements to magnetic fields below $850 \mathrm{G}$ to ensure low damping rates $\left(\gamma / \omega_{\perp}<0.01\right)$ and superfluid hydrodynamics.

At a given magnetic field, a set of measurements on the sloshing and compression modes typically takes a few hours. To minimize uncertainties from slow drifts and day-to-day variations we always took the sloshing mode reference measurement right before or after the compression mode data. By repeating measurements under identical settings we found a typical remaining fractional uncertainty for the normalized compression mode frequencies of $5 \times 10^{-3}$, which is about 2-3 times larger than the fit errors of individual measurements.

In Fig. 3 we show our final results on the normalized compression mode frequency in the BEC-BCS crossover. The two theory curves [19] correspond to the equation of state from mean-field BCS theory (lower curve) and the one from quantum Monte-Carlo calculations (upper curve). Our data confirm the quantum Monte-Carlo predictions and rule out the mean-field BCS theory. In the

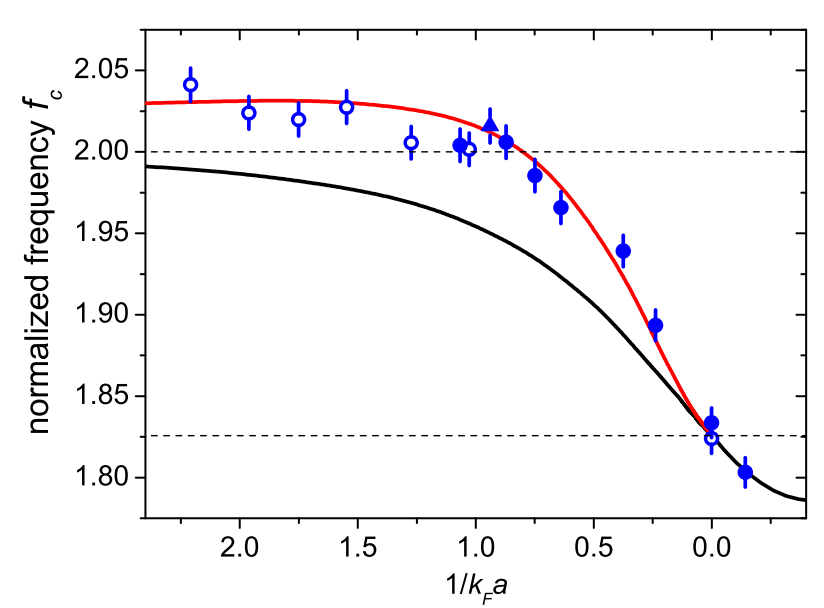

FIG. 3: Normalized compression mode frequency $f_{c}$ versus interaction parameter $1 / k_{F} a$. The experimental data include the small corrections for trap ellipticity and anharmonicity and can thus be directly compared to theory in the limit of an elongated harmonic trap with cylindrical symmetry. The open and filled circles refer to the measurements listed in Table \ for $\omega_{\perp} / 2 \pi \approx 290 \mathrm{~Hz}$ and $590 \mathrm{~Hz}$, respectively. The error bars indicate the typical scatter of the data points. The filled triangle shows a zero-temperature extrapolation of the measurements displayed in Fig. 44 The theory curves refer to mean-field BCS theory (lower curve) and QMC calculations (upper curve) and correspond to the data presented in Ref. [19]. The horizontal dashed lines indicate the values for the BEC limit $\left(f_{c}=2\right)$ and the unitarity limit $\left(f_{c}=\sqrt{10 / 3}=1.826\right)$.

strongly interacting $\mathrm{BEC}$ regime $\left(1 / k_{F} a \gtrsim 1\right)$ our data are well above the value of 2 . This highlights the presence of the long-sought beyond-mean-field effects 17] in collective modes of a strongly interacting gas [18, 26].

We finally address the question how non-zero temperatures influence the compression mode frequency. At unitarity, a recent experiment 27] has found small frequency upshifts with temperature. For a BEC, however, theory [28] predicts temperature-induced down-shifts, which compete with the up-shifts from beyond-mean-field effects. We have performed a set of measurements on temperature shifts in the strongly interacting BEC regime $\left(1 / k_{F} a=0.94\right)$. Before exciting the collective oscillation, the evaporatively cooled gas was kept in the recompressed trap for a variable hold time of up to $1.5 \mathrm{~s}$. During this time residual heating by inelastic processes slowly increased the temperature, which we observed as a substantial increase of damping with time. The damping rate $\gamma$ thus serves us as a very sensitive, but uncalibrated thermometer [8, 27]. Fig. [4 where we plot the normalized compression mode frequency versus damping rate, clearly shows a temperature-induced down-shift. We note that previous measurements in the strongly interacting BEC regime [9, 11] were performed at relatively large damping rates in the range between 0.05 and 0.1 , where frequency down-shifts are significant.

With our new knowledge on systematic frequency 


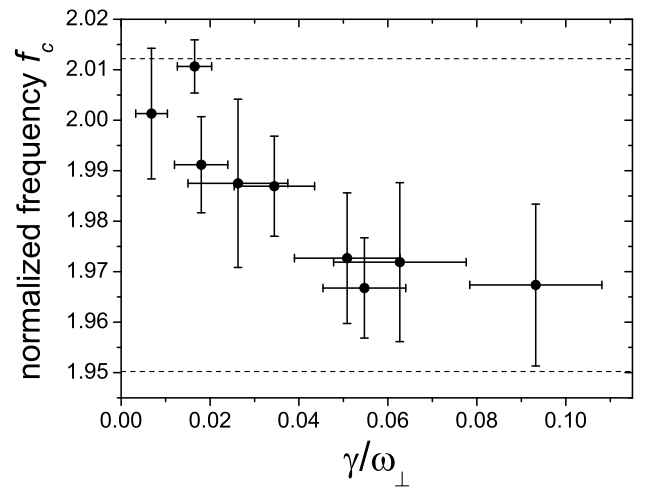

FIG. 4: Normalized compression mode frequency $f_{c}$ versus damping rate for $1 / k_{F} a=0.94\left(U_{0}=7.3 \mu \mathrm{K}\right)$. The error bars represent $1 \sigma$ fit uncertainties. The dashed lines indicate the zero-temperature values predicted by QMC calculations (upper line) and mean-field BCS theory (lower line).

shifts in collective mode measurements, let us comment on the previous experiments performed in Innsbruck [9] and at Duke University [8, 11]. We have reanalyzed our old data on the radial compression mode and identified a previously undetermined ellipticity of $\epsilon \approx 0.2$ as the main problem in our data interpretation [29]. The fact that we had normalized the compression mode frequency to the vertical trap frequency $\left(\omega_{c} / \omega_{y}\right)$ led to a substantial down shift in the hydrodynamic regime, but not in the collisionless regime. We furthermore believe that significant temperature shifts were present in the previous collective mode experiments. In particular for the strongly interacting BEC regime, temperature shifts in our old data on the axial mode 9] and the Duke data on the radial mode [11] provide a plausible explanation for these measurements being closer to the predictions of mean-field BCS theory than to the more advanced QMC results.

In conclusion, our work shows that collective modes allow for precision tests of many-body theories in strongly interacting quantum gases. In future experiments, the observation of collective oscillation modes will serve as a powerful tool to investigate strongly interacting superfluids in a more general context, e.g. in mixtures of fermionic quantum gases.

We warmly thank S. Stringari for stimulating our interest in collective modes and for many useful discussions. We thank G. Astrakharchik for providing us with the theoretical data for Fig. 3 and R. Danielian for assistance in the experiments. We acknowledge support by the Austrian Science Fund (FWF) within SFB 15 (project part 21). S.R. is supported within the Doktorandenprogramm of the Austrian Academy of Sciences. C.C. acknowledges travel support from the NSF-MRSEC program under DMR-0213745.
[1] K. M. O'Hara et al., Science 298, 2179 (2002).

[2] T. Bourdel et al., Phys. Rev. Lett. 91, 020402 (2003).

[3] S. Jochim et al., Science 302, 2101 (2003); M. Greiner, C. A. Regal, and D. S. Jin, Nature 426, 537 (2003); M. W. Zwierlein et al., Phys. Rev. Lett. 91, 250401 (2003).

[4] M. Bartenstein et al., Phys. Rev. Lett. 92, 120401 (2004).

[5] C. A. Regal, M. Greiner, and D. S. Jin, Phys. Rev. Lett. 92, 040403 (2004);

[6] M. W. Zwierlein et al., Phys. Rev. Lett. 92, 120403 (2004).

[7] T. Bourdel et al., Phys. Rev. Lett. 93, 050401 (2004).

[8] J. Kinast et al., Phys. Rev. Lett. 92, 150402 (2004).

[9] M. Bartenstein et al., Phys. Rev. Lett. 92, 203201 (2004).

[10] C. Chin et al., Science 305, 1128 (2004).

[11] J. Kinast, A. Turlapov, and J. E. Thomas, Phys. Rev. A 70, 051401(R) (2004).

[12] M. W. Zwierlein et al., Nature 435, 1047 (2005).

[13] G. B. Partridge et al., Phys. Rev. Lett. 95, 020404 (2005).

[14] D. M. Eagles, Phys. Rev. 186, 456 (1969); A. J. Leggett, in Modern Trends in the Theory of Condensed Matter, edited by A. Pekalski and R. Przystawa (Springer Verlag, Berlin, 1980); P. Nozières and S. Schmitt-Rink, J. Low Temp. Phys. 59, 195 (1985); J. R. Engelbrecht, M. Randeria, and C. Sá de Melo, Phys. Rev. B 55, 15153 (1997).

[15] P. Pieri, L. Pisani, and G. C. Strinati, Phys. Rev. B 72, 012506 (2005).

[16] G. E. Astrakharchik, J. Boronat, J. Casulleras, and
S. Giorgini, Phys. Rev. Lett. 93, 200404 (2004).

[17] T. D. Lee and C. N. Yang, Phys. Rev. 105, 1119 (1957); T. D. Lee, K. Huang, and C. N. Yang, Phys. Rev. 106, 1135 (1957).

[18] S. Stringari, Europhys. Lett. 65, 749 (2004); H. Heiselberg, Phys. Rev. Lett. 93, 040402 (2004); H. Hu, A. Minguzzi, X.-J. Liu, and M. P. Tosi, Phys. Rev. Lett. 93, 190403 (2004); A. Bulgac and G. F. Bertsch, Phys. Rev. Lett. 94, 070401 (2005); Y. E. Kim and A. L. Zubarev, Phys. Rev. A 70, 033612 (2004); N. Manini and L. Salasnich, Phys. Rev. A 71, 033625 (2005).

[19] G. E. Astrakharchik, R. Combescot, X. Leyronas, and S. Stringari, Phys. Rev. Lett. 95, 030404 (2005).

[20] M. Bartenstein et al., Phys. Rev. Lett. 94, 103201 (2005).

[21] A. Altmeyer, $\mathrm{PhD}$ thesis, Univ. Innsbruck, in preparation.

[22] F. Chevy et al., Phys. Rev. Lett. 88, 250402 (2002).

[23] We obtain $r_{\text {rms }}$ from in-situ measurements of axial profiles [4], using the relation $r_{\mathrm{rms}}=2 \omega_{z}^{2} / \omega_{r}^{2} z_{\mathrm{rms}}$.

[24] S. Stringari, private communication.

[25] J. Kinast, PhD thesis, Duke University (2006).

[26] L. Pitaevskii and S. Stringari, Phys. Rev. Lett. 81, 4541 (1998).

[27] J. Kinast, A. Turlapov, and J. E. Thomas, Phys. Rev. Lett. 94, 170404 (2005).

[28] S. Giorgini, Phys. Rev. A 61, 063615 (2000).

[29] A. Altmeyer et al., cond-mat/0611285 